\title{
THE DUALS OF HARMONIC BERGMAN SPACES
}

\author{
CHARLES V. COFFMAN AND JONATHAN COHEN \\ (Communicated by Clifford J. Earle, Jr.)
}

\begin{abstract}
In this paper we show that for $\Omega$, a starlike Lipschitz domain, the dual of the space of harmonic functions in $L^{p}(\Omega)$ need not be the harmonic functions in $L^{q}(\Omega)$, where $1 / p+1 / q=1$. We show that, as a consequence, the harmonic Bergman projection for $\Omega$ need not extend to a bounded operator on $L^{p}(\Omega)$ for all $1<p<\infty$. The duality result is a partial answer to a question of Nakai and Sario [9] posed initially in the Proceedings of the London Mathematical Society in 1978. We treat the duality question as a biharmonic problem, and our result follows from the failure of uniqueness for the biharmonic Dirichlet problem in domains with sharp intruding corners.
\end{abstract}

\section{INTRODUCTION}

In this paper we give a partial answer to a question posed by Nakai and Sario concerning the duals of harmonic Bergman spaces. Their question, initially posed in the Proceedings of the London Mathematical Society $[9$, p. 345] was whether, for $1<p<\infty, \Omega \subset R^{n}$, the dual of the space of harmonic functions in $L^{p}(\Omega)$ is the space of harmonic functions in $L^{q}(\Omega)$, where $1 / p+1 / q=1$, $1<p, q<\infty$. When the answer is affirmative, we will say that the duality property holds (for the given value of $p$ ). We note that every domain has the property for $p=2$, and domains which are bounded and have a sufficiently smooth boundary have the property for every $p$.

In 1981 Nakai and Sario [10] showed that the duality property can fail for $\Omega=\mathbf{R}^{2} \backslash\left\{z_{1}, z_{2}, \ldots, z_{n}\right\}$, and one of the authors subsequently discovered that duality also fails for the punctured disk.

In this paper we show that there is a $p$ between 1 and 2 and a Lipschitz domain with an intruding corner for which the duality property fails. We show that, as a consequence, there are Lipschitz domains on which the harmonic Bergman projection does not extend to a bounded operator on a full range of $p$ 's between 1 and $\infty$.

Our approach is to reduce the duality question to a biharmonic problem. We first show that the duality property is equivalent to the direct sum decomposition

Received by the editors June 30, 1989.

1980 Mathematics Subject Classification (1985 Revision). Primary 31A25, 35J40.

The first author was supported in part by NSF grant DMS-87040530; the second author was supported in part by a DePaul University development grant. 
of $L^{p}(\Omega)$ into the sum of the harmonic $L^{p}$ functions and the $L^{p}$ closure of the Laplacians of $C_{0}^{\infty}(\Omega)$ functions. The duality then fails if the spaces have nontrivial intersection or if the sum fails to exhaust all of $L^{p}$.

The nontrivial intersection is seen to occur when uniqueness fails for the biharmonic Dirichlet problem. The direct sum fails to exhaust $L^{p}$ when the nonhomogeneous biharmonic problem with zero Dirichlet data does not have a solution with Laplacian in $L^{p}$. In either case the counterexamples are constructed from biharmonic functions in wedges with angles greater than $180^{\circ}$ and with zero boundary data on the edges of the wedge.

\section{Motivation AND BACKGROUND}

For $\Omega \subset \mathbf{R}^{2}$ and $1<p<\infty$, we define

$$
L_{h}^{p}(\Omega)=\left\{u \in L^{p}(\Omega): \Delta u=0 \text { in } \Omega\right\} .
$$

Nakai and Sario showed that $L_{h}^{p}(\Omega)$ is a Banach space (see [9, p. 344] for details). They point out that $L_{h}^{2}(\Omega)^{*}=L_{h}^{2}(\Omega)$ and ask for which domains the following property holds:

$$
L_{h}^{p}(\Omega)^{*}=L_{h}^{q}(\Omega), \quad \text { where } 1<p<\infty, 1 / p+1 / q=1 .
$$

Property (1.2) will be called the duality property. Domains $\Omega$ for which (1.2) holds will be said to have the duality property.

Our approach to the duality question arises from a second consideration. Weyl's lemma (see Morrey, [8, p. 42]) implies that $L_{h}^{q}(\Omega)=M^{p}(\Omega)^{\perp}$, where $M^{p}(\Omega)$ is the $L^{p}(\Omega)$ closure of $\Delta C_{0}^{\infty}(\Omega)$. Since $L^{p}(\Omega)$ is reflexive and $M^{p}(\Omega)$ is a closed subspace of $L^{p}(\Omega), M^{p}(\Omega)^{\perp \perp}=M^{p}(\Omega)$. Hence $L_{h}^{q}(\Omega)^{*}=$ $L^{p}(\Omega) / M^{p}(\Omega)$. For the case $p=q=2$, the fact that $L_{h}^{2}(\Omega)=M^{2}(\Omega)^{\perp}$ implies the orthogonal decomposition $L^{2}(\Omega)=L_{h}^{2}(\Omega) \oplus M^{2}(\Omega)$. It is then natural to ask for which domains the direct sum decomposition

$$
L^{p}(\Omega)=L_{h}^{p}(\Omega)+M^{p}(\Omega)
$$

holds. The importance of the decomposition (1.3) in this paper is that it is equivalent to the duality property (1.2). To see this, note that since $L^{p}$ is reflexive and $M^{p}$ is closed, we have

$$
L_{h}^{q}(\Omega)^{*}=L^{q}(\Omega)^{*} / L_{h}^{q}(\Omega)^{\perp}=L^{p}(\Omega) / M^{p}(\Omega)^{\perp \perp}=L^{p}(\Omega) / M^{p}(\Omega) .
$$

It follows immediately that if the decomposition (1.3) holds, then $L_{h}^{p}(\Omega)=$ $L_{h}^{q}(\Omega)^{*}$. Conversely, if $L_{h}^{p}(\Omega)=L_{h}^{q}(\Omega)^{*}$, then the map $u \rightarrow u+M^{p}(\Omega)$ is a Banach space isomorphism from $L_{h}^{p}(\Omega)$ to the quotient space $L^{p}(\Omega) / M^{p}(\Omega)$. This fact together with (1.4) enables us to conclude that the decomposition (1.3) holds.

For $1 / p+1 / q=1, L_{h}^{p}(\Omega) \subset L_{h}^{q}(\Omega)^{*}$ in the sense that every element $\varphi \in$ $L_{h}^{p}(\Omega)$ gives rise to a linear functional $\lambda_{\varphi}$ on $L_{h}^{q}(\Omega)$ given by $\lambda_{\varphi}(f)=\iint_{\Omega} \varphi f$. 
We let $i_{p}$ denote the inclusion map of $L_{h}^{p}(\Omega)$ into $L_{h}^{q}(\Omega)^{*}$. Then $\Omega$ has the duality property when $i_{p}$ is bijective.

For $1<q<2$, applying Holder's inequality twice tells us that

$$
\iint \varphi u \leq\|\varphi\|_{p}\|u\|_{q} \leq\|\varphi\|_{p}\|u\|_{2}|\Omega|^{1 / q-1 / 2}
$$

where $|\Omega|$ denotes the measure of $\Omega$. Hence for $\Omega$ of finite measure, $L_{h}^{q}(\Omega)^{*}$ $\subset L_{h}^{2}(\Omega)^{*}$. Finally, as noted earlier, $L_{h}^{2}(\Omega)=L_{h}^{2}(\Omega)^{*}$ in the sense that every element in the dual of $L_{h}^{2}(\Omega)$ can be represented uniquely as integration against a function in $L_{h}^{2}(\Omega)$. We conclude that for $p>2$ and $\Omega$ a set of finite measure,

$$
L_{h}^{p}(\Omega) \subset L_{h}^{q}(\Omega)^{*} \subset L_{h}^{2}(\Omega)^{*}=L_{h}^{2}(\Omega)
$$

This factorization of the inclusion $L_{h}^{p}(\Omega) \subset L_{h}^{2}(\Omega)$ implies that when $\Omega$ is bounded or of finite measure, $i_{p}$ is injective for $p>2$. Since the adjoint of $i_{p}$ is $i_{q}$ where $1 / p+1 / q=1$, we have that the duality property holds for $\Omega$ if $i_{p}$ is surjective for $p>2$.

From now on, we assume the domain to be bounded or at least of finite measure. We proceed formally, showing how the solution of a biharmonic Dirichlet problem suffices to show that $i_{p}$ is surjective. For $1 / p+1 / q=1$, $p>2$, and $\lambda \in L_{h}^{q}(\Omega)^{*}$, there exists $\varphi \in L^{p}(\Omega)$ satisfying

$$
\lambda(u)=\iint_{\Omega} \varphi(x) u(x) d x \quad \text { for all } u \in L_{h}^{q}(\Omega)
$$

We want to be able to choose a $\varphi \in L^{p}(\Omega)$ which satisfies (1.6) and is harmonic. First we find a $w$ satisfying $\Delta w=\varphi$. If $\Delta^{2} w=0$, then $\varphi$ is harmonic and we're done. If not, let $v$ be another function, regular enough to permit integration by parts.

$$
\begin{aligned}
\lambda(u)= & \iint_{\Omega} \varphi u \\
= & \iint_{\Omega}(\Delta w-\Delta v) u+\iint_{\Omega} \Delta v \cdot u \\
= & \iint_{\partial \Omega}(\partial w / \partial n-\partial v / \partial n) u-(w-v) \partial u / \partial n \\
& +\iint_{\Omega}(w-v) \Delta u+\iint_{\Omega} \Delta v \cdot u .
\end{aligned}
$$

If $\Delta v \in L_{h}^{p}(\Omega)$ and the other integrals are zero, then $\lambda$ can be represented by an element in $L_{h}^{p}(\Omega)$. In other words, $\lambda$ can be represented by an element 
in $L_{h}^{p}(\Omega)$ if we can find $v$ satisfying

$$
\begin{aligned}
\Delta^{2} v & =0 \quad \text { in } \Omega, \\
\left.v\right|_{\partial \Omega} & =\left.w\right|_{\partial \Omega}, \\
\partial v / \partial n & =\partial w / \partial n,
\end{aligned}
$$

and

$$
\Delta v \in L^{p}(\Omega),
$$

or, more precisely, we want $v-w \in W_{0}^{2, p}(\Omega)$.

Since by assumption $p>2$, the inclusion map $i_{p}: L_{h}^{p}(\Omega) \rightarrow L_{h}^{q}(\Omega)^{*}$ is 1 to 1 , and we can conclude that the duality property holds.

It is important to note that the duality property is reflexive in the sense that $L_{h}^{q}(\Omega)^{*}=L_{h}^{p}(\Omega)$ if and only if $L_{h}^{p}(\Omega)^{*}=L_{h}^{q}(\Omega)$. On the other hand, the existence theory for the biharmonic equation is not reflexive on Lipschitz domains. That is, the theory for data in $L^{p}(\partial \Omega)$ for $1<p<2$ is not like the theory for $2<p<\infty$. (See Dahlberg, Kenig, and Verchota [4, pp. 130-133] for details in the range $1<p<2$ in $\mathbf{R}^{2}$ and Pipher and Verchota [11, 12] for proof of existence in the range $2-\varepsilon<p<\infty$ in $\mathbf{R}^{3}$.) This suggests possible problems on domains with sharp intruding corners.

Some positive results are known for the duality property. For bounded smooth domains, the property follows from the biharmonic equation approach using standard results in elliptic theory (see Lions and Magenes [7]). One can then easily prove the following:

Proposition (1.10). For $\Omega$ a smooth bounded domain the following are equivalent:

(1.11) $i_{p}$ is surjective.

(1.12) For $u \in W^{2, p}$ there is a biharmonic $w \in W^{2, p}$ with $w \rightarrow u \in W_{0}^{2, p}$.

For bounded $C^{1}$ domains in $\mathbf{R}^{2}$, Soleve' ev states [13] that the duality property holds. For the unit disk there is a substantial literature on the duals of harmonic Bergman spaces including weighted versions and those dealing with the range $0<p<\infty$. (See Coifman and Rochberg [3], Ligowcka [5] for a sample of these results.)

For negative results, Nakai and Sario [9] showed that $\mathbf{R}^{2} \backslash\left\{z_{1}, \ldots, z_{n}\right\}$ could be a domain not having the duality property. This follows from the fact that for $\Omega=\mathbf{R}^{2} \backslash\left\{z_{1}, \ldots, z_{n}\right\}, L_{h}^{p}(\Omega)$ consists of functions of the form $f(z)=$ $\sum_{j=1}^{n} c_{j} \ln \left|z-z_{j}\right|$. By choosing the $c_{j}$ 's cleverly, one can show that for $1<p<$ $2, L_{h}^{p}(\Omega)$ has dimension 0 but $L_{h}^{q}(\Omega)$ has dimension at least 1 for $q>2$. By a dimension argument, $L_{h}^{p}(\Omega)^{*}=L_{h}^{q}(\Omega)$ is then impossible.

Another interesting example is that of the punctured disk $D_{0}=D \backslash\{0\}=$ $\left\{z \in \mathbf{R}^{2}: 0<|z|<1\right\}$, which also does not satisfy the duality property. This example is more complicated than the plane minus a finite set of points, since 
the space $L_{h}^{p}\left(D_{0}\right)$ is infinite-dimensional. However, in $D_{0},(2 r-1 / r) \cos \theta$ and $(2 r-1 / r) \sin \theta$ are in $L_{h}^{p}\left(D_{0}\right)$ for $1<p<2$ and additionally satisfy

$$
\begin{aligned}
& \int_{0}^{2 \pi} \int_{0}^{1}(2 r-1 / r) \cos \theta \cdot u(r, \theta) r d r d \theta=0 \\
& \int_{0}^{2 \pi} \int_{0}^{1}(2 r-1 / r) \sin \theta \cdot u(r, \theta) r d r d \theta=0
\end{aligned}
$$

for all $u \in L_{h}^{q}\left(D_{0}\right)$ where $q>2$. In other words, for $1 / p+1 / q=1,1<$ $p<2,2<q<\infty$, every element in $L_{h}^{q}\left(D_{0}\right)$ annihilates $(2 r-1 / r) \cos \theta$ and $(2 r-1 / r) \sin \theta$. But by the Hahn-Banach theorem there exists a function $g \in L^{p}\left(D_{0}\right)^{*}=L^{q}\left(D_{0}\right)$ which doesn't vanish on $(2 r-1 / r) \sin \theta$. As an element of $L_{h}^{p}\left(D_{0}\right)^{*}, g$ cannot be represented by a harmonic function because this would violate (1.13).

It is also true that $\Delta\left(r \ln r-r^{3} / 2+r / 2\right) \sin \theta=(3 / r-4 r) \sin \theta \in L_{h}^{p}\left(D_{0}\right) \cap$ $M^{p}\left(D_{0}\right)$, and so property (1.3) is seen to fail as well.

Another interesting property of $D_{0}$ is that the projection operator which maps $L^{2}\left(D_{0}\right) \rightarrow L_{h}^{2}\left(D_{0}\right)$ does extend to a bounded operator on $L^{p}\left(D_{0}\right)$, since an orthonormal basis for $L_{h}^{2}\left(D_{0}\right)$ can be obtained from the usual basis for $L_{h}^{2}(D)$ by adjoining the element $\ln r$.

In this paper we show that there is a simply connected Lipschitz domain for which the duality property fails for some $p$ between 1 and 2 . Furthermore, we show that the Bergman projection must also fail to be bounded on $L^{p}$ for this particular $p$. The last result is the oppositve situation from that of the punctured disk where the boundary point at the origin allows for the duality property to fail without affecting the boundedness of the Bergman projection.

\section{THE FAILURE OF THE DUALITY PROPERTY}

In this section we show how the duality property can fail on a Lipschitz domain with a sharp intruding corner. We construct our counterexample through the solution of a biharmonic equation. However, rather than showing that $i_{p}$ is not surjective for some $p>2$, we show that $i_{p}$ can fail to be injective for $1<p<2$. From the point of view of the biharmonic Dirichlet problem this amounts to showing a failure of uniqueness.

Dahlberg, Kenig, and Verchota [4] have shown that there exists a bounded Lipschitz domain $\Omega \subset \mathbf{R}^{2}$ and a biharmonic function $u \in \Omega$ whose Dirichlet data is zero. $\left(\Delta^{2} u=0\right.$ in $\Omega, u=0$ and $\partial u / \partial n=0$ on $\left.\partial \Omega\right)$. One would like to conclude, then, that $\Delta u$ is a nontrivial element in $M^{p}(\Omega) \cap L_{h}^{p}(\Omega)$. Unfortunately, the construction yields a function $u \in W^{3 / 2,2}(\Omega)$. Hence one cannot conclude that $\Delta u \in L^{p}(\Omega)$. In fact, one has $\Delta u \in W^{-1 / 2,2}(\Omega)$. Instead we use the existence theorem (Grisvard [6, p. 302]) to construct a biharmonic function $w$ with $w$ and its normal derivative vanishing on the boundary of $\Omega$ and for which $\Delta w \in L^{p}(\Omega)$ where $1<p<2$. It then follows easily that $\Delta w \in L_{h}^{q}(\Omega)^{\perp}$ and so $L_{h}^{q}(\Omega)^{*} \neq L_{h}^{p}(\Omega)$. 
Lemma 5.1 of Dahlberg, Kenig, and Verchota [4] proves that there exist constants $\gamma, \lambda, \alpha$ with $0<\lambda<1 / 2, \pi / 2<\alpha<\pi$ and a function $\psi(r, \theta)=$ $r^{\lambda}(\sin \lambda \theta-\gamma \sin (\lambda-2) \theta)$ satifying

$$
\begin{aligned}
\Delta^{2} \psi(r, \theta) & =0 \quad r>0,-\alpha<\theta<\alpha, \\
\psi(r, \pm \alpha) & =0, \\
\psi_{\theta}(r, \pm \alpha) & =0 .
\end{aligned}
$$

For $A$ a bounded neighborhood of the corner, $\Delta \psi \in L^{p}(A)$, provided $p<$ $2 /(2-\lambda)$ and $\Delta \psi \notin L^{p}(A)$ when $p \geq 2 /(2-\lambda)$. In particular, for $\lambda<1 / 2$, $\Delta u \notin L^{2}(A)$. (In fact $\Delta u \notin L^{2}$ for $2>2 /(2-\lambda)$ if and only if $\lambda<1$ ). On the other hand, for any $0<\lambda<1 / 2$, there is a $p>1$ for which $p<2 /(2-\lambda)$. We point out that Grisvard shows $([6$, Lemma 7.3 .2 .4$, p. 338$]$ that it is necessary that $\alpha>\pi / 2$.

Consider the domain $\Omega$ whose boundary $\partial \Omega=\{(r, \pm \alpha): 0 \leq r \leq 1\} \cup S$ where $S$ is $C^{\infty}$ and completes the domain so that $\Omega$ is starlike. (e.g., $S$ can be a circular arc smoothed out when it meets the edge of the sector so $\Omega$ looks like Pacman).

Let $\varphi \in C^{\infty}(\Omega)$ with $\varphi=0$ in a small neighborhood of 0 and $\varphi \equiv 1$ outside a small neighborhood of the origin which does not intersect $S$. By Grisvard [6, Lemma 7.1, p. 302], there exists $w$ satisfying $\Delta^{2} w=\Delta^{2} \varphi \psi$, with $w$ and its normal derivative vanishing on the boundary and with the regularity $\Delta w \in L^{2}(\Omega)$; or more precisely, $w \in W_{0}^{2,2}(\Omega)$. Set $v=\psi+(w-\varphi \psi)$. Then $\Delta^{2} v=\Delta^{2} \psi+\Delta^{2} w-\Delta^{2} \varphi \psi=0,\left.v\right|_{\partial \Omega}=0, \partial v /\left.\partial n\right|_{\partial \Omega}=0$. Again, more precisely, since obviously $\psi-\varphi \psi \in W_{0}^{2, p}(\Omega)$, we have $v \in W_{0}^{2, p}(\Omega)$. On the one hand $\Delta \psi \in L^{p}(\Omega)$ for some $1<p<2$. On the other hand, $\Delta \psi \notin L^{2}(\Omega)$. Hence we conclude that $v \neq 0$, since $\Delta(w-\varphi \psi) \in L^{2}(\Omega)$ and $\Delta \psi \notin L^{2}(\Omega)$.

As indicated earlier, we now have a function $v$ with zero biharmonic Dirichlet data and with $\Delta v \in L_{h}^{p}(\Omega)$. For any $u \in L_{h}^{p}(\Omega), 1 / p+1 / q=1$,

$$
\iint_{\Omega} \Delta v \cdot u=\int_{\partial \Omega} \partial v / \partial n \cdot u-\int_{\partial \Omega} v \cdot \partial u / \partial n+\iint_{\Omega} v \cdot \Delta u=0
$$

Alternatively, the same follows from the fact that $v \in W_{0}^{2, p}(\Omega)$. But $\Delta v \neq 0$, and so there exists a function $g \in L^{q}(\Omega)$ with $\iint g \cdot \Delta v \neq 0$. By (2.2), $g$ cannot be harmonic. In other words, we have proved the existence of a linear functional on $L_{h}^{p}(\Omega)$ which can not be represented by a harmonic function. This means that the duality property fails.

\section{THE FAILURE OF THE HARMONIC PROJECTION IN $L^{p}$.}

Let $\Omega$ be a bounded domain in $\mathbf{R}^{2}$. The harmonic kernel function is the reproducing kernel function for the space $L_{h}^{2}(\Omega)$. A detailed description of reproducing kernel spaces can be found in [1], but for our purposes the following short explanation will suffice. 
For $x \in \Omega$, the solid mean-value theorem and Schwartz inequality imply that pointwise evaluation of an $L_{h}^{2}(\Omega)$ function at $x$ is a bounded linear functional on $L_{h}^{2}(\Omega)$. Hence, by the Riesz representation theorem, for each $x \in \Omega$, there is a unique, symmetric $K(x, z)$ satifying $\Delta_{z} K(x, z)=0$ for all $z \in \Omega$, $\iint_{\Omega}|K(x, z)|^{2} d z<\infty$, and $u(x)=\iint_{\Omega} K(x, z) u(z) d z$ for all $u \in L_{h}^{2}(\Omega)$. (Note that $d z$ represents area measure in $\Omega$ ).

As remarked earlier, it follows from Weyl's lemma that $L^{2}(\Omega)=L_{h}^{2}(\Omega) \oplus$ $M^{2}(\Omega)$. This guarantees that the opeator $T f(x)=\iint_{\Omega} K(x, z) f(z) d z$ is a bounded operator on $L^{2}(\Omega)$. In fact, $T$ is the projection from $L^{2}(\Omega)$ onto $L_{h}^{2}(\Omega)$.

In this section we consider the question of determining domains on which the operator $T$ extends to a bounded operator on $L^{p}(\Omega)$ for $1<p<\infty$.

We recall that $D_{0}$ denotes the punctured unit disk. Now we let $K_{D}(x, z)$ denote the kernel function for the full unit disk and $K_{0}(x, z)$ the kernel function for the punctured unit disk. Clearly,

$$
K_{0}(x, z)=K_{D}(x, z)+C_{1} \ln \left(C_{2}|x|\right) \ln \left(C_{2}|z|\right) .
$$

Letting $T_{0} f(x)=\iint_{\Omega} K_{0}(x, z) f(z) d z$, we see that $T_{0}$ extends to a bounded operator on $L^{p}(\Omega)$ for $1<p<\infty$. This means that it is possible for the harmonic Bergman projection to extend to a bounded operator on all of $L^{p}$ but for the duality property to fail.

We next consider the starlike domain $\Omega_{p}$ described in $\S 2$ and make a small translation so that the domain is starlike with respect to the origin. We let $K_{P}(x, z)$ denote the kernel function for the domain $\Omega_{P}$. If the operator $T_{P} f(x)=\iint_{\Omega} K_{P}(x, z) f(z) d z$ extends to a bounded operator on all of $L^{p}\left(\Omega_{P}\right)$, then by duality $\iint_{\Omega_{P}}\left|K_{P}(x, z)\right|^{q} d z<\infty$ for $1 / p+1 / q=1$ and for all $x \in \Omega$. Hence as a function of $z, K_{P}(x, z) \in L_{h}^{q}\left(\Omega_{P}\right)$ for all $x \in \Omega_{P}$.

We recall from $\S 2$ the existence of a biharmonic function $v$ with zero Dirichlet data for which $\Delta v \in L^{p}\left(\Omega_{P}\right) \backslash L^{2}\left(\Omega_{P}\right)$. We let $w(x)=\Delta v(x)$ and define $w_{t}(x)=w(t x)$. Then $w_{t}$ is harmonic and bounded in $\Omega_{P}$ for $0<t<1$. In particular, $w_{t} \in L_{h}^{2}\left(\Omega_{P}\right)$. Hence

$$
\begin{aligned}
w_{t}(x) & =\iint_{\Omega_{P}} K_{P}(x, z) w_{t}(z) d z \\
& =\iint_{\Omega_{P}} K_{P}(x, z)\left(w_{t}(z)-w(z)\right) d z,
\end{aligned}
$$

since, as we observed in $\S 2, w \in L_{h}^{q}\left(\Omega_{P}\right)^{\perp}$. If the Bergman operator $T_{p}$ is bounded on $L^{p}\left(\Omega_{P}\right)$, we get

$$
\left\|w_{t}\right\|_{p} \leq c\left\|w_{t}-w\right\|_{p}=o(1) \quad \text { as } t \rightarrow 1 .
$$

This would imply that $\|w\|_{p}=0$, which we know is false. So for this example, the starlike nature of the domain allows us to infer the failure of the boundedness of the Bergman operator in $L^{p}\left(\Omega_{P}\right)$ from the failure of the duality property 
in $\Omega_{P}$. We point out that this is in sharp contrast to the punctured disk where duality fails, but the Bergman operator is bounded on $L^{p}\left(D_{0}\right)$.

Concluding remark. The above argument shows that a starlike domain $\Omega$ has the duality property for a given $p$ if and only if the (harmonic) Bergman operator is bounded on $L^{p}(\Omega)$. A slightly more involved argument shows that this remains true for domains with continuous boundary in the sense of Grisvard $[6$, p. 5], i.e., equivalent domains with the uniform segment property.

\section{BIBLIOGRAPHY}

1. N. Aronszajn, Theory of reproducing kernels, Trans. Amer. Math. Soc. 68 (1950), 337-404.

2. D. Bekolle, Projections sur des espaces de fonctions holomorphes dans des domaines plans, Canad. J. Math. 38 (1986), 127-157.

3. R. Coifman and R. Rochberg, Representation theorems for holomorphic functions in $L^{p}$, Asterisque 77, (1980), 11-66.

4. B. Dahlberg, C. Kenig, and G. Verchota, The Dirichlet problem for the biharmonic equation in a Lipschitz domain, Ann. Inst. Fourier (Grenoble) 36 (1986), 109-135.

5. E. Ligowcka, On the duality and interpolation for spaces of polyharmonic functions, Studia Math. T.87.1 (1987), 23-32.

6. P. Grisvard, Elliptic boundary value problems on non-smooth domains, Pittman, 1983.

7. J. L. Lions and E. Magenes, Problemi ai limiti non omogenei (V), Ann. Scuola Norm. Sup. 16 (1962), 1-44.

8. C. B. Morrey, Multiple integrals in the calculus of variations, Springer, New York, 1966.

9. M. Nakai and L. Sario, Existence of biharmonic Green's functions, Proc. London Math. Soc. 36 (1978), 337-368.

10. __ Banach spaces of harmonic functions in $L_{p}$, Bull. Inst. Math. Acad. Sinica, 6 (1978), 415-417.

11. J. Pipher and G. Verchota, Area integral estimates for the biharmonic operator in Lipschitz domains, preprint.

12. ___ Biharmonic Dirichlet problem on Lipschitz domains in $\mathbf{R}^{3}$, preprint.

13. A. A. Solov' ev, $L^{p}$-estimates of integral operators associated with spaces of analytic and harmonic functions, Soviet Math. Dokl. 19 (1978), 764-768.

14. G. Verchota, The Dirichlet problem for the biharmonic equation in $C^{1}$ domains, Indiana Math. J. 36 (1987), 867-895.

Department of Mathematics, Carnegie Mellon University, Pittsburgh, Pennsylvania 15213

Mathematics Department, DePaul University, Chicago, Illinois 60614 